\title{
Corrigendum to Recommendation on Test Readiness Criteria for New Approach Methods in Toxicology: Exemplified for Developmental Neurotoxicity
}

\author{
Anna Bal-Price1, Helena T. Hogberg'2, Kevin M. Crofton ${ }^{3}$, Mardas Daneshian 4 , Rex E. FitzGerald5, \\ Ellen Fritsche 6 , Tuula Heinonen 7 , Susanne Hougaard Bennekou ${ }^{8}$, Stefanie Klima ${ }^{9}$, Aldert H. Piersma10, \\ Magdalini Sachana ${ }^{11}$, Timothy J. Shafer ${ }^{3}$, Andrea Terron ${ }^{12}$, Florianne Monnet-Tschudi ${ }^{5,13}$, Barbara Viviani ${ }^{14}$, \\ Tanja Waldmann ${ }^{9}$, Remco H. S. Westerink ${ }^{15}$, Martin F. Wilks ${ }^{5}$, Hilda Witters ${ }^{16}$, Marie-Gabrielle Zurich ${ }^{5,13}$ \\ and Marcel Leist 4,9 \\ ${ }^{1}$ European Commission, Joint Research Centre (EC JRC), Ispra, Italy; ${ }^{2}$ Center for Alternatives to Animal Testing (CAAT), Johns Hopkins University, \\ Baltimore, MD, USA; ${ }^{3}$ National Center for Computational Toxicology, US EPA, RTP, NC, USA; ${ }^{4}$ Center for Alternatives to Animal Testing, CAAT- \\ Europe, University of Konstanz, Konstanz, Germany; ${ }^{5}$ Swiss Centre for Human Applied Toxicology, SCAHT, University of Basle, Basle, Switzerland; \\ ${ }^{6}$ IUF - Leibniz Research Institute for Environmental Medicine \& Heinrich-Heine-University, Düsseldorf, Germany; ${ }^{7}$ Finnish Centre for Alternative \\ Methods (FICAM), University of Tampere, Tampere, Finland; ${ }^{8}$ Danish Environmental Protection Agency, Copenhagen, Denmark; ${ }^{9}$ In vitro Toxicology \\ and Biomedicine, Dept inaugurated by the Doerenkamp-Zbinden Foundation, University of Konstanz, Konstanz, Germany; ${ }^{10}$ RIVM, National Institute for \\ Public Health and the Environment, Bilthoven, and Institute for Risk Assessment Sciences, Utrecht University, Utrecht, The Netherlands; ${ }^{11}$ Organisation \\ for Economic Co-operation and Development (OECD), Paris, France; ${ }^{12}$ European Food Safety Authority, Parma, Italy; ${ }^{13}$ Department of Physiology, \\ University of Lausanne, Lausanne, Switzerland; ${ }^{14}$ Department of Pharmacological and Biomolecular Sciences, University of Milan, Milan, Italy; \\ ${ }^{15}$ Neurotoxicology Research Group, Institute for Risk Assessment Sciences (IRAS), Faculty of Veterinary Medicine, Utrecht University, Utrecht, \\ The Netherlands; ${ }^{16}$ VITO, Flemish Institute for Technological Research, Unit Environmental Risk and Health, Mol, Belgium
}

In this manuscript, which appeared in ALTEX 35, 306-352 (doi:10.14573/altex.1712081), the Acknowledgements should read:

\section{Acknowledgements}

This work was supported by the Doerenkamp-Zbinden Foundation, EFSA, the BMBF, JPI-NutriCog-Selenius, and it has received funding from the European Union's Horizon 2020 research and innovation programme under grant agreement No. 681002 (EU-ToxRisk).

doi:10.14573/altex.1904112

\section{Corrigendum to Essential Components of Methods Papers}

\author{
Marcel Leist 1,2,3 and Jan G. Hengstler 4,5 \\ ${ }^{1}$ In vitro Toxicology and Biomedicine, Dept inaugurated by the Doerenkamp-Zbinden Foundation, University of Konstanz, Konstanz, Germany; ${ }^{2}$ Konstanz \\ Research School Chemical Biology (KoRS-CB) \& Co-operative research training school 'Advanced in-vitro test systems for the analysis of cell-chemical \\ interactions in drug discovery and environmental safety (inviTe)', University of Konstanz, Konstanz, Germany; ${ }^{3}$ CAAT-Europe, University of Konstanz, \\ Konstanz, Germany; ${ }^{4}$ Leibniz Research Centre for Working Environment and Human Factors (IfADo), Technical University of Dortmund, Dortmund, \\ Germany; ${ }^{5}$ Archives of Toxicology, editor-in-chief, Heidelberg, Germany
}

In this manuscript, which appeared in ALTEX 35, 429-432 (doi:10.14573/altex.1807031), the Acknowledgements should read:

\section{Acknowledgements}

This work was supported by BMBF and DFG (KoRS-CB) grants, and it has received funding from the European Union's Horizon 2020 research and innovation programme under grant agreement No. 681002 (EU-ToxRisk).

doi:10.14573/altex.1904114 